\title{
Surfactant-controlled ink drying enables high-speed deposition of perovskite films for efficient photovoltaic modules
}

\author{
Yehao Deng ${ }^{1,2}$, Xiaopeng Zheng ${ }^{2}$, Yang Bai ${ }^{2}$, Qi Wang ${ }^{1,2}$, Jingjing Zhao ${ }^{2}{ }^{2}$ and Jinsong Huang $\oplus^{1,2 \star}$
}

\begin{abstract}
Novel photovoltaic technologies such as perovskites hold the promise of a reduced levelized cost of electricity, but the low-cost potential depends on the ability to scale-up solution-based deposition. So far, complex fluid dynamics have limited the solution deposition of uniform pinhole-free organic-inorganic perovskite thin films over large areas. Here, we show that very small amounts (tens of parts per million) of surfactants (for example, $L-\alpha$-Phosphatidylcholine) dramatically alter the fluid drying dynamics and increase the adhesion of the perovskite ink to the underlying non-wetting charge transport layer. The additives enable blading of smooth perovskite films at a coating rate of $180 \mathrm{~m} \mathrm{~h}^{-1}$ with root-mean-square roughness of $14.5 \mathrm{~nm}$ over $1 \mathrm{~cm}$. The surfactants also passivate charge traps, resulting in efficiencies over $20 \%$ for small-area solar cells. Fast blading in air of perovskite films results in stabilized module efficiencies of $15.3 \%$ and $14.6 \%$ measured at aperture areas of $33.0 \mathrm{~cm}^{2}$ and $57.2 \mathrm{~cm}^{2}$, respectively.
\end{abstract}

P erovskite solar cells (PSCs) have joined the high-efficiency photovoltaic league as the youngest member ${ }^{1-4}$. After demonstration of very high power conversion efficiency over $20 \%$ for small area devices in laboratories ${ }^{5-8}$, the next big question is whether the high efficiency lab cells can be translated to solar modules with minimal efficiency loss in industrial scale high-throughput production. One significant advantage of perovskite materials is their solution processing capability, which allows perovskite solar panels to be manufactured by scalable and low-cost vacuum-free processes. However, most of the reported efficient PSCs are still fabricated by spin-coating, a low-throughput process that limits cell size to about $1 \mathrm{~cm}^{2}$. Scalable coating methods for the perovskite layers have been sought in the past few years, such as spray-coating ${ }^{9-11}$, electrochemical deposition ${ }^{12}$, soft-cover deposition ${ }^{13,14}$, doctor-blade coating $^{15-19}$ and slot-die coating ${ }^{20-22}$. Among them, blade-coating is a continuous fabrication process and has been one of the most successful methods due to its simplicity and versatility, making it easily adopted in research laboratories ${ }^{16,18}$. Blade-coating was first introduced for PSC fabrication in 2015 with a highest reported device efficiency of $\sim 15.0 \%{ }^{16}$, and the efficiency was quickly increased to over $19.0 \%$ for small-area $\left(\sim 0.1 \mathrm{~cm}^{2}\right)$ solar cells by perovskite composition engineering ${ }^{18,23}$. In addition, blade-coating has close analogy to several industrial-level high-throughput coating techniques, such as slot-die coating and gravure coating, and thus can be translated into more sophisticated roll-to-roll coating. Despite the promising progress made for small-area devices by blade-coating, a much larger variation of device performance for the bladed devices was generally observed than for spun devices. The non-uniformity of the bladed films makes it very challenging to fabricate efficient large area PSCs, while the reported efficiencies for spun larger area $\left(\sim 1 \mathrm{~cm}^{2}\right)$ perovskite devices are close to $20 \%{ }^{3,4}$. The non-uniformity of the bladed films may be caused by the inherent solution flow dynamics ${ }^{19,24}$, which is not present in spin-coating processes, because blade-coating is generally done at higher temperature than spin-coating. In addition, the charge transport layer underlying the perovskite film, such as poly(bis(4-phenyl) (2,4,6-trimethylphenyl) amine)(PTAA) and poly(3-hexylthiophene-2,5-diyl) (P3HT), while required to achieve high power conversion efficiency (PCE), impose a significant challenge for blade-coating of continuous perovskite films. Indeed, these underlying layers are typically non-wetting to perovskite solution, which reduces the adhesion of the perovskite solution to the underlying layer during the drying process.

In this work, we report that the addition of a very small amount of surfactant additive at the level of approximately 20 parts per million ( $\mathrm{ppm}$ ) into the perovskite ink solution can alter the solution flow dynamics and enhance the adhesion of the perovskite solution to very hydrophobic hole transport materials. Surfactants from a variety of categories were tested to be effective. The additives enable the coating of uniform perovskite films over large areas at a high blade-coating rate of 180 metres per hour $\left(\mathrm{m} \mathrm{h}^{-1}\right)$. The very small amount of surfactant additive has no detrimental effect to the optoelectronic properties of bladed perovskite films, and some rationally designed surfactants even passivate the charge traps in bladed perovskite films. The bladed device efficiencies reach $20.3 \%$ for a $0.075 \mathrm{~cm}^{2}$ cell and above $15 \%$ for modules with an aperture area over $30 \mathrm{~cm}^{2}$. Based on this fast, high-quality, scalable perovskitecoating technique, an annual production capacity of $236 \mathrm{MW}$ with a one-metre-wide roll-to-roll manufacturing line is predicted.

\section{Blade-coating speed and film deposition mode}

The blade-coating of perovskite film is illustrated in Fig. 1a. A blade swipes perovskite precursor solution (ink) over a pre-heated substrate (typically $\sim 70-145^{\circ} \mathrm{C}$ ). As solvent evaporates, the perovskite precursor material crystallizes into black solid perovskite films. The pre-heating of the substrates can speed up blade-coating and suppress the formation of needle-like structures in the perovskite films, because a fast evaporation of solvent may convert the ink directly into perovskites by skipping the needle-like intermediate phase ${ }^{16,21,25}$. We measured perovskite film thickness as a function of coating speed after coating perovskite precursor solution of $1 \mathrm{M}$ methylammonium 

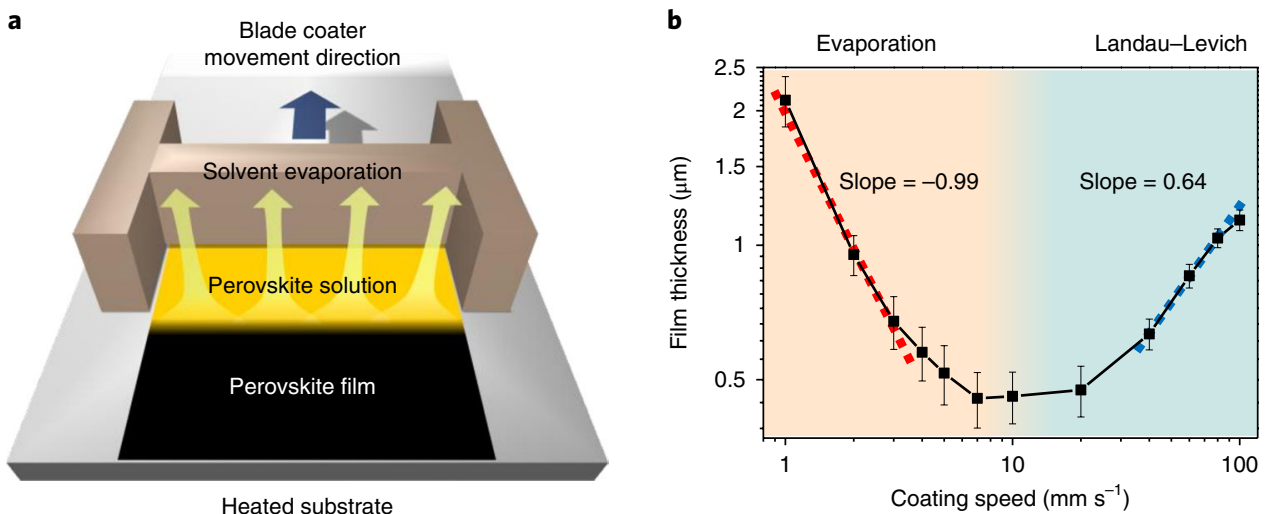

Fig. 1 | Blade-coating of perovskite films. a, Schematic illustration for the blade-coating of perovskite film in the Landau-Levich mode. $\mathbf{b}$, The perovskite

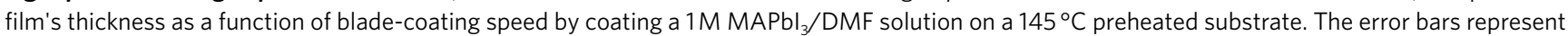

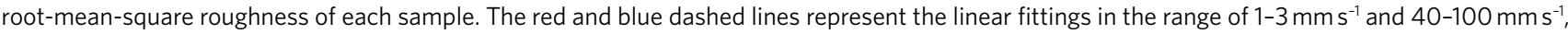
respectively, with the coefficient of determination being around 0.98 .

lead halide $\left(\mathrm{MAPbI}_{3}\right)$ in dimethylformamide (DMF) on glass substrates preheated at $145^{\circ} \mathrm{C}$. As is shown in Fig. $1 \mathrm{~b}$, the dependence of film thickness $(t)$ on the speed of swiping/coating $(v)$ would fall into two modes, which resembles that in a dip-coating process ${ }^{26}$. When the blading speed is below $4 \mathrm{~mm} \mathrm{~s}^{-1}$, the slope for the $\log (v)-$ $\log (t)$ plot is -0.99 . This indicates that blade-coating is in evaporation mode, where the ink dries right after the blade moves away from the ink surface, and thus evaporation of the ink meniscus between blade and substrate governs the solute deposition ${ }^{19,26}$. It has been demonstrated recently that high-quality perovskite films can be formed in this blading mode using high boiling solvent, yielding a device efficiency close to $20 \%$, however the very slow coating rate of 0.012 millimetres per second $\left(\mathrm{mm} \mathrm{s}^{-1}\right)$ limits its practical application ${ }^{19}$. When the blading speed is above $20 \mathrm{~mm} \mathrm{~s}^{-1}$, the slope is 0.64 , very close to the 0.66 predicted by Landau-Levich mode theory ${ }^{26}$. In Landau-Levich mode, the blading speed is much faster so that the as-coated thin ink layer is still wet right after blading. We are interested in developing high-quality perovskite films with Landau-Levich mode for fast PSC production.

In this study, the chosen blading speed was $50 \mathrm{~mm} \mathrm{~s}^{-1}$ or faster, which falls in the Landau-Levich region, aiming at realistic application. We indeed observed that the ink was still wet after moving away the blade at such a high speed. Due to the high surface tension and non-volatile property of most known perovskite solvents (Supplementary Table 1), the drying of a thin ink layer over a large-area substrate poses a challenge for depositing compact and uniform perovskite films with a thickness of 500-600 nm needed for high efficiency PSCs ${ }^{4}$. Big 'islands' about 10-200 micrometres across, assembled from many polycrystalline grains, are obtained in the bladed perovskite films ${ }^{16}$. There is often a large gap between one island and the next, which causes large leakage current or even failure of blade-coated PSCs. One typical example of a gap is shown in Fig. 2. It is noted that the same surface feature is also observed on the film fabricated by other solution-based scalable coating methods, such as slot-die coating ${ }^{13,21}$.

\section{Suppression of solution flow by surfactants}

Because the island structures only appear in films formed by scalable coating processes, they should be related to the different fluid flowing dynamics during drying of the perovskite solution. To find out how these islands and wide gaps form and thus to find a solution to avoid them, we set up a microscopy optical system to observe the in situ drying process of the perovskite solution right after blading. The optical system includes a fast optical microscope camera with a frame rate of 54 frames per second (fps). To slow down the drying process, $N$-methyl-2-pyrrolidone (NMP), which has a lower vapour pressure than other common perovskite solvents, was used as the solvent for $\mathrm{CH}_{3} \mathrm{NH}_{3} \mathrm{PbI}_{3}$. A coating temperature of $100^{\circ} \mathrm{C}$ was chosen to avoid fast ink drying considering that the boiling point of NMP is $202^{\circ} \mathrm{C}$. As shown in Fig. 2a and Supplementary Video 1, during drying of $1 \mathrm{M} \mathrm{MAPbI}_{3} / \mathrm{NMP}$ solution, particles with diameter of $\sim 1-3 \mu \mathrm{m}$ appeared in the solution at an early stage right after blading. These solid-state particles were identified to be the crystallized $\mathrm{MAPbI}_{3} \cdot \mathrm{NMP}$ intermediate phase by polarized optical microscopy and X-ray diffraction analysis (Supplementary Fig. 1). These $\mathrm{MAPbI}_{3} \cdot \mathrm{NMP}$ particles in the perovskite solution served as the tracer to show the solution flow direction. It was found that these particles kept nearly stationary within the first second after blading, and then started to move when a perovskite island directly crystallized from the ink (which bypasses the intermediate phase stage, as discussed in Supplementary Discussion following Supplementary Fig. 1) at approximately $1.00 \mathrm{~s}$ after blading. The moving directions for four of the particles at different locations are highlighted in Fig. 2a, showing that they all moved towards the perovskite island. By stacking 30 of the video frames from $1.00 \mathrm{~s}$ to $2.00 \mathrm{~s}$ into one image (Fig. 2b), the track of each particle is clearly revealed. Figure $2 b$ shows that these particles moved towards the perovskite island from all directions. The directional movement of particles continued till the formation of the perovskite island completed when the ink dried at $5.00 \mathrm{~s}$ after blading (Fig. 2a).

This observation suggests that there are microscale fluid flows moving towards perovskite islands during the drying of the perovskite ink after blading, which is illustrated in Fig. 2e by both a plane view and a cross-section view. The transport of perovskite solute by the flows toward the centre of the islands left partially filled or empty gaps between the islands. Almost the same ink drying processes and final morphologies were observed when we used other solvents such as (dimethyl sulfoxide) DMSO or DMF at the blading temperatures of $100-145^{\circ} \mathrm{C}$ (Supplementary Fig. 2). Therefore, the solution flow dynamics should be general for perovskite inks at a wide blading temperature range, though in many cases intermediate phase particles might not be observed due to the quick conversion of solution to perovskite crystals.

The microscale fluid flows observed should have a driving force, which is speculated to be induced by evaporation of solvent at the periphery of the perovskite islands. To suppress the flows for more uniform perovskite films, we proposed adding surfactants into the perovskite solution. As is well known, surfactant molecules contain both hydrophilic and hydrophobic functional groups. They can selfassemble on the surface of a solution with the hydrophobic group 


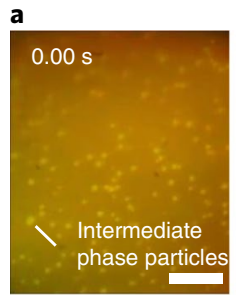

c
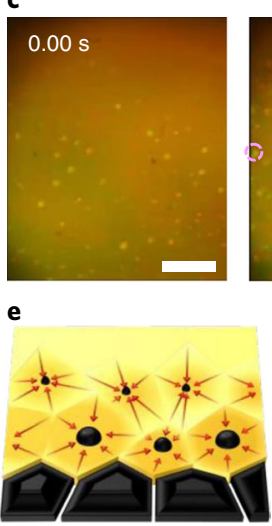

Solution flow
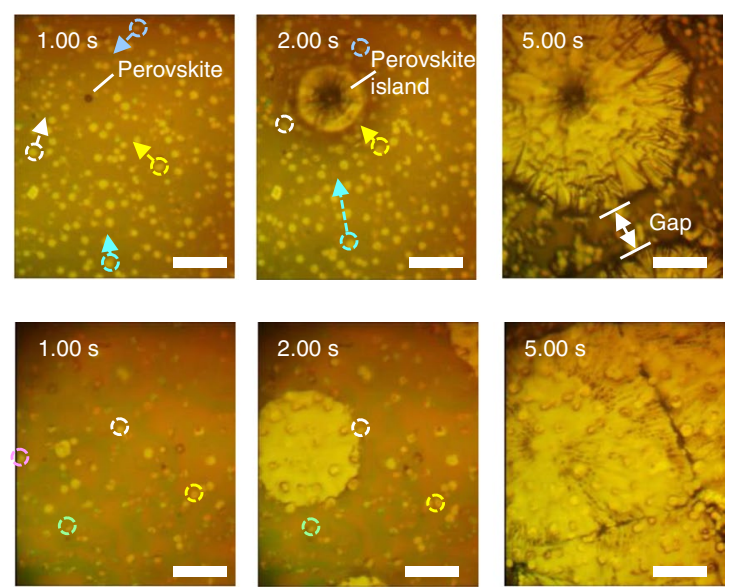

f
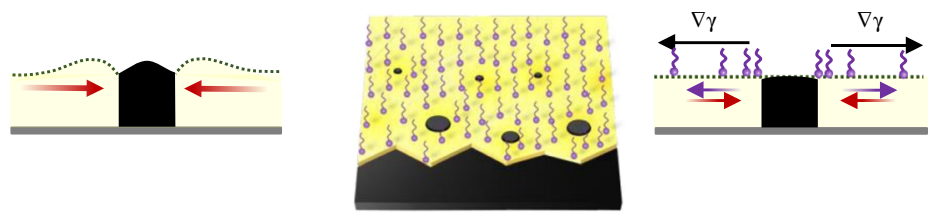

d
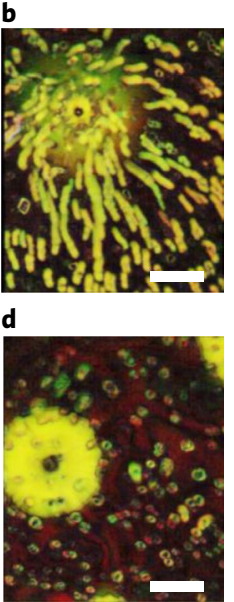

- - Hydrophobic

$\int$ - - Hydrophilic

Fig. 2 | Solution flow dynamics and its suppression by surfactants. a, In situ microscopy observation of the ink layer drying dynamics. Several intermediate phase particles are highlighted with dashed circles of different colour, and their movement directions are indicated by arrows. $\mathbf{b}$, Stacked video frames (Supplementary Video 1) from 1.00 s to 2.00 s to show the tracks of perovskite intermediate phase particles without LP surfactants. c, The ink layer drying dynamics in the presence of LP surfactant at a concentration of $\sim 20$ ppm. Similarly, several intermediate phase particles are highlighted and they remain stationary. d, Stacked video frames (Supplementary Video 2) from $1.00 \mathrm{~s}$ to $2.00 \mathrm{~s}$ to show the tracks of perovskite intermediate phase particles in the presence of LP surfactants. The scale bars in all figures are $20 \mu \mathrm{m}$. e, Schematic illustration for the directional microscale solution flow towards perovskite island during ink drying. $\mathbf{f}$, Schematic illustration for the suppressed solution flow dynamics in the presence of surfactant. $\nabla \gamma$, surface tension gradient.

pointing to air to reduce the surface tension of the solution, as illustrated in Fig. 2f. The reduction in surface tension was evidenced by the reduced contact angle of perovskite solution droplets on nonwetting substrate on addition of surfactants (Supplementary Fig. 3). When the directional flows transport surfactants to the periphery of perovskite islands, a surfactant concentration gradient is established with increasing concentration from solution to the island periphery, resulting in a decreasing surface tension gradient in the same direction (Fig. 2f). Consequently, a 'Marangoni flow ${ }^{27}$ driven by a surfactant-induced surface tension gradient is formed, flowing from island periphery to solution. The Marangoni flow counteracts the original flow, resulting in a completely suppressed fluid flow in the drying perovskite solution (Fig. 2f).

Several types of surfactant were tested to assist perovskite bladecoating, including L- $\alpha$-phosphatidylcholine (LP), polyethylene glycol sorbitan monostearate (Tween 60), sodium dodecyl sulfate (SDS) and didodecyldimethylammonium bromide (DDAB), which are amphoteric, non-ionic, anionic and cationic surfactants, respectively. Their molecular structures are shown in Supplementary Fig. 4. From top-view scanning electron microscopy (SEM) we found that all of them can suppress the island structure, though the degree of effectiveness varied (Fig. 3a-e). Among these surfactants, LP, which is an amphoteric surfactant, was chosen for optimization, because of its capability to passivate charge traps in hybrid perovskites with the charged quarteramonium ions ${ }^{28}$. When only $\sim 20$ ppm of LP was added, the directional movements of intermediate-phase particles toward perovskite islands disappeared, as shown in Fig. 2c and Supplementary Video 2. Stacking the video frames from $1.00 \mathrm{~s}$ to $2.00 \mathrm{~s}$ reveals no tracks of the motion of the particles
(Fig. 2d). It should be noted that the in situ microscopy study of Fig. 2 is done using NMP as solvent and a lower coating temperature of $100^{\circ} \mathrm{C}$, while the SEM images of Fig. 3 are all from coating with $0.8 \mathrm{M} \mathrm{MAPbI}_{3} / \mathrm{DMF}$ solution at $145^{\circ} \mathrm{C}$, which was optimized for device fabrication. Compared to the control sample with many island boundaries and gaps (Fig. 3a), the film with added LP has no such structure (Fig. 3b). The control film had a thickness variation from $1.4 \mu \mathrm{m}$ to $0 \mu \mathrm{m}$ from the island centre to the edge, which is a consequence of microflow that transports perovskite solute to the centre, resulting in the depletion of solute at the edge, that is, a gap (Fig. 3a). In striking contrast, the film bladed with LP had a uniform thickness of $\sim 0.65 \mu \mathrm{m}$ (Fig. $3 \mathrm{~b}$ ). The photographic image for the blade-coated films in Fig. 3 f clearly shows that the films with LP surfactant are much smoother than the control films. The surface roughness profiling of the film bladed with LP additive over a $1 \mathrm{~cm}$ length scale presented in Fig. 3h gives a root-mean-square roughness of $14.5 \mathrm{~nm}$, which is one order of magnitude smaller than that of the control film (Fig. 3g). For a completed device, any pin-holes in the perovskite layer would increase leakage current (dark current $)^{29}$. The compactness of the film with surfactant was evidenced by a very small leakage current density below $100 \mathrm{nA} \mathrm{cm}^{-2}$ at $-0.4 \mathrm{~V}$ bias for a $1 \mathrm{~cm}^{2}$ PSC (Supplementary Fig. 5), which is comparable to spin-coated perovskite devices with smaller area ${ }^{29}$.

\section{Wetting improvement to hydrophobic substrates}

De-wetting of perovskite solutions is another challenge that prevents the deposition of uniform perovskite films by blading, especially on indium tin oxide (ITO) substrates covered with hydrophobic poly(bis(4-phenyl) (2,4,6-trimethylphenyl) amine (PTAA), 

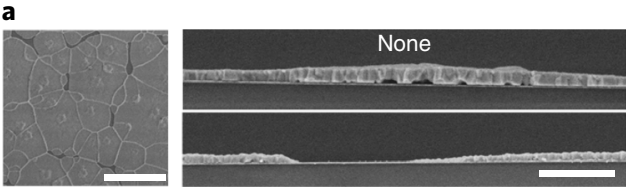

b
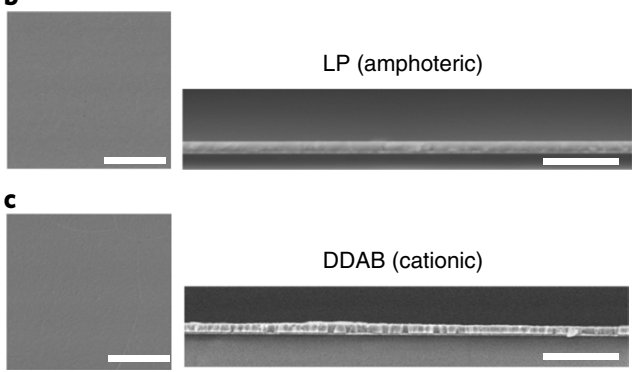

d
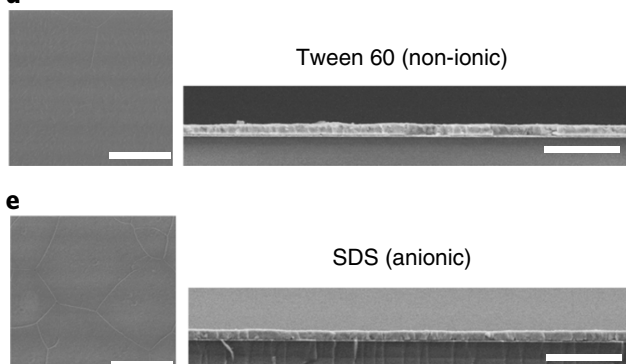

SDS (anionic)

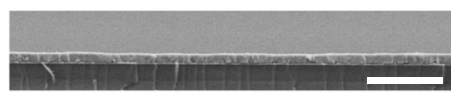

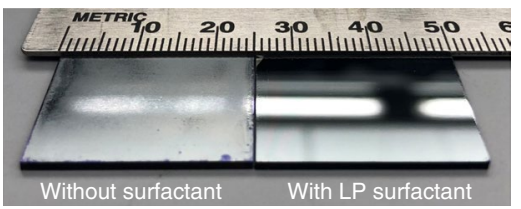
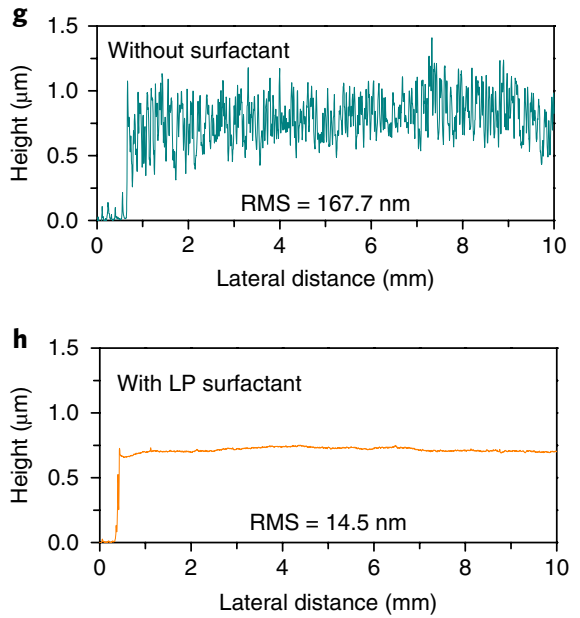

Fig. 3 | Morphological characterization of blade-coated perovskite films with surfactants. a-e, Plane view and cross-sectional SEM images of bladecoated perovskite films without surfactant and with LP, DDAB, Tween 60 and SDS, respectively. Scale bars for plane view and cross-sectional SEM images are $50 \mu \mathrm{m}$ and $5 \mu \mathrm{m}$, respectively. f, A photographic image of blade-coated perovskite films without and with LP surfactant. $\mathbf{g}$,h, Surface roughness profiling of blade-coated perovskite films without and with LP surfactant, respectively.

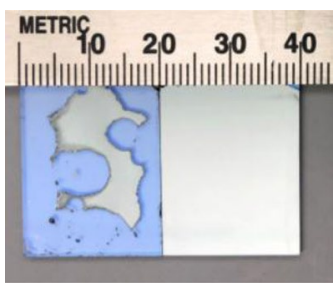

Hydrophobic surface

d

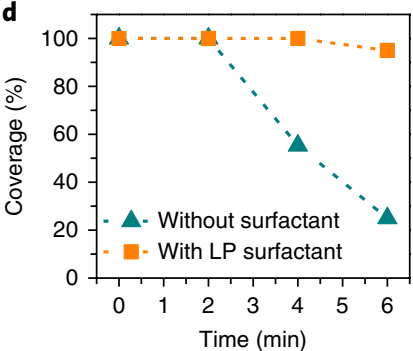

b

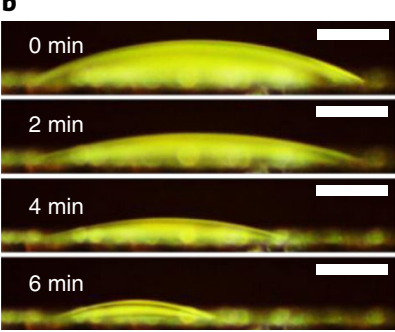

e

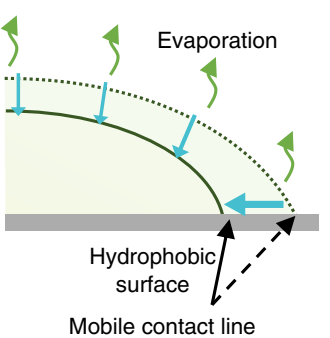

c

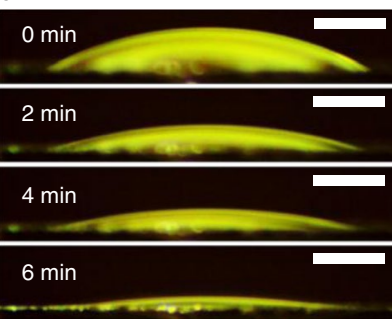

f

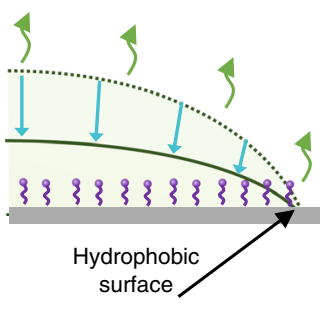

Pinned contact line

Fig. 4 | Wetting improvement of perovskite ink onto hydrophobic substrates by surfactants. a, Blade-coated perovskite films on hydrophobic substrates without (left) and with (right) LP surfactant. b,c, The drying processes of perovskite ink droplets $\left(\sim 0.5 \mu l, 0.125 \mathrm{M} \mathrm{MAPbl}_{3} / \mathrm{DMF}\right)$ on hydrophobic substrates under ambient conditions without and with LP surfactant, respectively. The scale bars are $0.5 \mathrm{~mm}$. $\mathbf{d}$, The evolution of coverage of perovskite droplets during drying. e, Schematic illustration for the shrinkage of the ink droplet drying on a hydrophobic substrate. $\mathbf{f}$, Schematic illustration for the surfactant pinning effect.

which is needed for high efficiency devices ${ }^{30}$. The perovskite solution first spread over the substrate by the blade, but shrunk quickly during drying, leaving most of the area uncovered after drying
(Fig. 4a, left). To better understand the process, we monitored the drying of a perovskite ink droplet $\left(\sim 0.5 \mu \mathrm{l}, 0.125 \mathrm{MMAPbI}_{3} / \mathrm{DMF}\right)$ on a hydrophobic substrate under ambient conditions (Fig. 4b). During 
the solvent evaporation, the droplet volume decreased, and the contact line (the edge of the droplet) moved with the coverage (defined by the projected area of the droplet on the substrate during drying versus that at the beginning) reducing to $25 \%$ (Fig. $4 \mathrm{~d}$ ). The nonwetting problem was solved when LP surfactant was blended into the perovskite solution (Fig. 4c). The coverage of the droplet remained almost $100 \%$ during the whole drying period. The contact line was strongly 'pinned' onto the substrate. The pinning effect is commonly observed with hydrophilic, but not hypdrophobc, substrates ${ }^{31}$. Therefore, it indicates that surfactant effectively modified the ink/ substrate interface and improved the affinity of perovskite ink to hydrophobic substrate, as is schematically illustrated in Fig. 4e, $\mathrm{f}^{32,33}$. The bladed perovskite films with LP surfactant additive could easily achieve full coverage on the PTAA/ITO/glass substrate, as shown in Fig. $4 \mathrm{a}$, right. The wetting improvement was also observed with other types of surfactant, as is shown in Supplementary Fig. 6.

\section{Blade-coated perovskite device characterization}

The performances of PSCs made by the surfactant-assisted bladecoating are presented in Fig. 5. The device structure is ITO/PTAA/ $\mathrm{MAPbI}_{3} /$ fullerene $\left(\mathrm{C}_{60}\right)$ /bathocuproine $(\mathrm{BCP}) / \mathrm{Cu}$. A device with a small area of $7.5 \mathrm{~mm}^{2}$ has a short circuit current density $\left(J_{\text {sc }}\right)$ of $22.4 \mathrm{~mA} \mathrm{~cm}^{-2}$, an open circuit voltage $\left(V_{\mathrm{oc}}\right)$ of $1.12 \mathrm{~V}$ and a fill factor (FF) of $81.0 \%$ under one sun simulated illumination, giving a PCE of $20.3 \%$ (Fig. $5 \mathrm{a}$ ). The integrated $J_{\mathrm{sc}}$ of $22.6 \mathrm{~mA} \mathrm{~cm}^{-2}$ from external quantum efficiency agrees with $J_{\text {sc }}$ from $J-V$ scanning measurement (Supplementary Fig. 7). Devices fabricated with Tween 60 and $\mathrm{DDAB}$ as surfactant additives showed lower efficiencies, around 18.0\% (Supplementary Fig. 8), but still higher than our previous best results based on $\mathrm{MAPbI}_{3}$ without surfactant ${ }^{17}$. This confirms
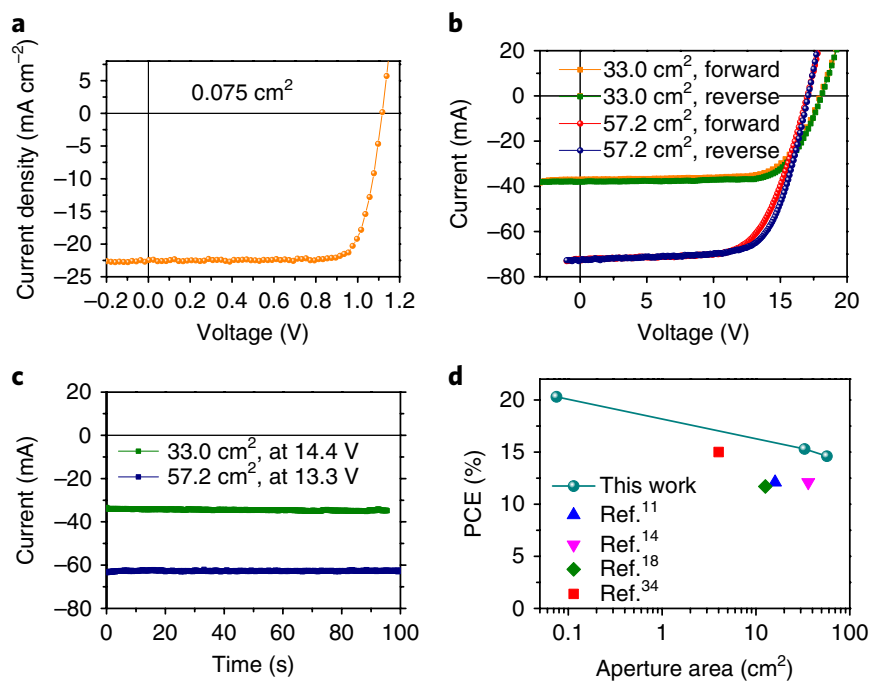

e

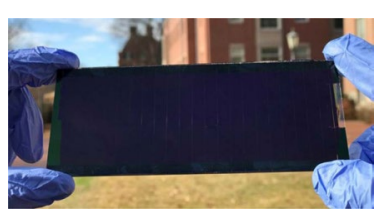

f

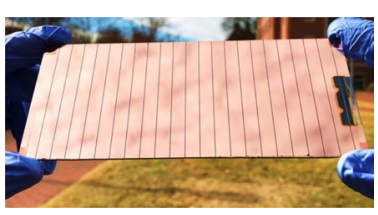

Fig. 5 | Characterization of surfactant-assisted blade-coated PSC and modules. a, Current density-voltage $(J-V)$ scanning of a $0.075 \mathrm{~cm}^{2}$ surfactant-assisted blade-coated PSC. b,c, I-V scanning curves and stabilized photocurrent output at the maximum power point of modules with aperture areas of $33.0 \mathrm{~cm}^{2}$ and $57.2 \mathrm{~cm}^{2}$. d, Power conversion efficiency of PSCs as a function of aperture area from this work and recent representative reports ${ }^{11,14,18,34}$. e,f, Photographic image of a perovskite solar module viewed from the glass side (left) and top electrode side (right). that there are two functions of the LP surfactant: improving film quality by its surfactant nature and passivating perovskite defects by its functional groups ${ }^{28}$.

The surfactant-assisted blading method was also evaluated for fabricating large-area photovoltaic modules because of its scalability. To achieve a high-efficiency module, the entire large-area film is required to be defect-free. Even a single pin-hole may cause the shunt of a sub-cell, which dramatically reduces the performance of the module. Therefore, a module that retains the high efficiency of small-area cells is an excellent demonstration of the high quality of the large-area perovskite film. Photos of a perovskite solar module fabricated on a $6 \times 15 \mathrm{~cm}^{2}$ glass substrate are shown in Fig. 5e,f. Each sub-cell in the module has a width of $0.65 \mathrm{~cm}$, in which the nonworking area for connecting sub-cells in series (classic p1-p2-p3 configuration) is $0.043 \mathrm{~cm}$ wide (Supplementary Fig. 9). Therefore, the geometric fill factor, the ratio of active area to the aperture area, is $93.4 \%$, which is among the best values for perovskite solar modules $^{34}$. The detailed module design of sub-cell dimension and number of sub-cells are included in Supplementary Table 2. The photocurrents of modules with aperture area of $33.0 \mathrm{~cm}^{2}$ and $57.2 \mathrm{~cm}^{2}$ show minor hysteresis with different bias scanning directions under one sun simulated illumination (Fig. 5b). To provide a better evaluation of as-fabricated modules with respect to regular perovskite single cells, the module PCE, FF and sub-cell equivalent $J_{\mathrm{sc}}$ and $V_{\mathrm{oc}}$ based on $I-V$ curves in Fig. $5 \mathrm{~b}$ are included in Supplementary Table 2 as well. The stabilized photocurrents of the two modules at their respective maximum power output point are shown in Fig. 5c, giving stabilized PCEs of $15.3 \%$ and $14.6 \%$, respectively. The PCE, FF and sub-cell equivalent $V_{\mathrm{oc}}$ and $J_{\mathrm{sc}}$ statistics of eight modules with an aperture area of $\sim 57.2 \mathrm{~cm}^{2}$ fabricated from four batches are presented in Supplementary Fig. 10, showing good reproducibility for this method. It should be noted that perovskite films were coated outside of clean rooms and in air. The high module efficiency was confirmed by an external laboratory, the Solar Power Lab at Arizona State University. The results are shown in Supplementary Fig. 11. The measured module, with an aperture area of $57.8 \mathrm{~cm}^{2}$, had PCE of $14.9 \%$ from reverse scan and $14.7 \%$ from forward scan. Figure $5 \mathrm{~d}$ summarizes the device efficiencies of the bladed cell and modules with different aperture areas. It shows that the bladed devices maintain relatively high efficiencies when the aperture area is increased by two orders of magnitude, confirming the excellent quality of the perovskite films over the large area formed by this method. Figure $5 \mathrm{~d}$ also includes the reported best efficiencies of perovskite modules with different aperture areas fabricated by different methods ${ }^{11,14,18,34}$, which shows that the modules reported in this work have high efficiencies. Considering a coating speed of $50 \mathrm{~mm} \mathrm{~s}^{-1}\left(180 \mathrm{~m} \mathrm{~h}^{-1}\right)$, and a PCE of $15.0 \%$ for the module, the surfactant-assisted scalable coating should in principle allow an annual production capacity of $236 \mathrm{MW}$ with a one-metre-wide roll-to-roll manufacturing line. A preliminary stability study of the as-fabricated module shows no degradation of the PCE after over 20 days of storage in inert atmosphere (Supplementary Fig. 12).

\section{Conclusion}

We have demonstrated that surfactant additives of $\sim 20 \mathrm{ppm}$ in perovskite ink can significantly improve the blade-coating quality of perovskite films at a high coating speed of $180 \mathrm{~m} \mathrm{~h}^{-1}$. PSCs and large-area modules with high efficiencies were demonstrated. The mechanistic studies reveal that surfactant effectively suppresses the solution flow dynamics in the drying ink layer and yields fullcoverage coating by improving the adhesion of perovskite ink to hydrophobic substrates. Some surfactants could further improve device performance because of their passivation effect. Therefore, the surfactants could be a kind of general additive in perovskite inks for improving perovskite film quality in various scalable fabrication methods. 


\section{Methods}

Materials. All the materials were from Sigma Aldrich unless otherwise specified. Methylammonium iodide (MAI) was synthesized according to our previous method $^{16}$. The CAS (Chemical Abstracts Service) numbers and product codes for all surfactants used in this study are: LP, 8002-43-5 (CAS), P3556 (product code); didodecyldimethylammonium bromide, 3282-73-3, 359025; polyethylene glycol sorbitan monostearate (TWEEN 60), 9005-67-8, P1629; sodium dodecyl sulfate, 151-21-3, 436143. PTAA was purchased from Sigma Aldrich and Xi'an Polymer Light Technology Corp.

Device fabrication. ITO/glass substrates were patterned by etching with $\mathrm{HCl}$ and zinc powder and washed with detergent, deionized water, isopropanol and acetone sequentially before drying in an oven at $60^{\circ} \mathrm{C}$ overnight. $5 \mathrm{mg} \mathrm{m}^{-1}$ of PTAA/toluene solution was blade-coated on UV-ozone treated ITO/glass substrate at $20 \mathrm{~mm} \mathrm{~s}^{-1}$. The gap between blade-coater and substrate was $100 \mu \mathrm{m}$ and the solution amount was $2.5 \mu \mathrm{cm}^{-1}(2.5 \mu \mathrm{l}$ for every $1 \mathrm{~cm}$ of substrate width). Then the PTAA layer was annealed at $100^{\circ} \mathrm{C}$ for $10 \mathrm{~min}$. Perovskite layer blade-coating was adjusted from a previous method ${ }^{16,17}$. Here, surfactants were added into the perovskite precursor solution before use. For coating the best performing devices with LP surfactant, the concentration of $\mathrm{MAPbI}_{3} / \mathrm{DMF}$ was $0.8 \mathrm{M}\left(496 \mathrm{mg} \mathrm{ml}^{-1}\right)$ and that of LP/DMF was $0.25 \mathrm{mM}\left(0.2 \mathrm{mg} \mathrm{ml}^{-1}\right)$. The other surfactants used for mechanism study were in the same molar concentration. Then $5-7 \mu \mathrm{lcm}^{-1}$ of the precursor solution was swiped linearly by a film applicator with a gap between blade-coater and substrate of $50-100 \mu \mathrm{m}$. The coating temperature was $145^{\circ} \mathrm{C}$ and coating speed was $50 \mathrm{~mm} \mathrm{~s}^{-1}$ for best performing devices. After coating, the perovskite film was annealed for $10 \mathrm{~min}$ at $100^{\circ} \mathrm{C}$. The PTAA and perovskite layers blade-coatings were done in air. Then $30 \mathrm{~nm}$ of $\mathrm{C}_{60}, 6 \mathrm{~nm}$ of BCP and $100 \mathrm{~nm}$ of copper were thermally evaporated sequentially to complete the devices.

To fabricate perovskite solar modules, the ITO electrode, PTAA/perovskite/ $\mathrm{C}_{60} / \mathrm{BCP}$ stacking layer, and $\mathrm{Cu}$ electrode were ablated by an excimer laser scriber (Resonetics Rapid X250) sequentially to form a p1-p2-p3 pattern as shown in Supplementary Fig. 9. The design of laser scribing experiment is referred to recent publications $\mathrm{s}^{35,36}$. The widths are $50 \mu \mathrm{m}, 100 \mu \mathrm{m}$ and $150 \mu \mathrm{m}$ for ITO, PTAA/ perovskite/ $\mathrm{C}_{60} / \mathrm{BCP}$ and $\mathrm{Cu}$ electrode, respectively, which is limited by our current laser scribing system.

Device characterization. The $J-V$ measurement of devices was performed with a Keithley 2400 Source-Meter under simulated AM 1.5 G irradiation produced by a xenon-lamp-based solar simulator (Oriel Sol3A, Class AAA Solar Simulator). The light intensity was calibrated by a silicon reference cell (Newport 91150V-KG5). The scan rate was $0.2 \mathrm{~V} \mathrm{~s}^{-1}$ for small area device and $1 \mathrm{~V} \mathrm{~s}^{-1}$ for modules. The delay time was $10 \mathrm{~ms}$. There was no preconditioning before measurement. The steadystate PCE was measured by applying the bias voltage at maximum power output point obtained by $I-V$ scanning and recording the photocurrent. The external quantum efficiency (EQE) spectra were obtained by a Newport QE measurement kit by focusing a monochromatic light beam with wavelength from $300 \mathrm{~nm}$ to $900 \mathrm{~nm}$ onto the devices. More information on the solar cell testing can be found in the Reporting Summary. SEM images were taken by a Quanta 200 FEG environmental scanning electron microscope. The XRD pattern was obtained with a Bruker-AXS D8 Discover Diffractometer. The surface roughness profiling of blade-coated perovskite films was measured with a Dektak XT profiler.

Reporting Summary. Further information on experimental design is available in the Nature Research Reporting Summary linked to this article.

Data availability. The data that support the plots within this paper and other findings of this study are available from the corresponding author upon reasonable request.

Received: 2 September 2017; Accepted: 11 April 2018; Published online: 14 May 2018

\section{References}

1. Yablonovitch, E. Lead halides join the top optoelectronic league. Science 351, 1401 (2016).

2. Yang, Y. \& You, J. Make perovskite solar cells stable. Nature 544, 155-156 (2017)

3. Li, X. et al. A vacuum flash-assisted solution process for high-efficiency large-area perovskite solar cells. Science 353, 58-62 (2016).

4. Tan, H. et al. Efficient and stable solution-processed planar perovskite solar cells via contact passivation. Science 355, 722-726 (2017).

5. Yang, W. S. et al. Iodide management in formamidinium-lead-halide-based perovskite layers for efficient solar cells. Science 356, 1376-1379 (2017).

6. Son, D.-Y. et al. Self-formed grain boundary healing layer for highly efficient $\mathrm{CH}_{3} \mathrm{NH}_{3} \mathrm{PbI}_{3}$ perovskite solar cells. Nat. Energy 1, 16081 (2016).

7. Zhao, D. et al. Low-bandgap mixed tin-lead iodide perovskite absorbers with long carrier lifetimes for all-perovskite tandem solar cells. Nat. Energy 2, 17018 (2017).
8. Eperon, G. E. et al. Perovskite-perovskite tandem photovoltaics with optimized band gaps. Science 354, 861-865 (2016).

9. Barrows, A. T. et al. Efficient planar heterojunction mixed-halide perovskite solar cells deposited via spray-deposition. Energy Environ. Sci. 7, 2944-2950 (2014)

10. Bag, S., Deneault, J. R. \& Durstock, M. F. Aerosol-jet-assisted thin-film growth of $\mathrm{CH}_{3} \mathrm{NH}_{3} \mathrm{PbI}_{3}$ perovskites - a means to achieve high quality, defect-free films for efficient solar cells. Adv. Energy Mater. 7, 1701151 (2017).

11. Kim, J. et al. Overcoming the challenges of large-area high-efficiency perovskite solar cells. ACS Energy Lett. 2, 1978-1984 (2017).

12. Chen, H., Wei, Z., Zheng, X. \& Yang, S. A scalable electrodeposition route to the low-cost, versatile and controllable fabrication of perovskite solar cells. Nano Energy 15, 216-226 (2015).

13. Ye, F. et al. Soft-cover deposition of scaling-up uniform perovskite thin films for high cost-performance solar cells. Energy Environ. Sci. 9, 2295-2301 (2016).

14. Chen, $\mathrm{H}$. et al. A solvent-and vacuum-free route to large-area perovskite films for efficient solar modules. Nature 550, 92-95 (2017).

15. Kim, J. H., Williams, S. T., Cho, N., Chueh, C.-C. \& Jen, A. K. Y. Enhanced environmental stability of planar heterojunction perovskite solar cells based on blade-coating. Adv. Energy Mater. 5, 1401229 (2015).

16. Deng, Y. et al. Scalable fabrication of efficient organolead trihalide perovskite solar cells with doctor-bladed active layers. Energy Environ. Sci. 8, 1544-1550 (2015).

17. Deng, Y., Dong, Q., Bi, C., Yuan, Y. \& Huang, J. Air-stable, efficient mixed-cation perovskite solar cells with cu electrode by scalable fabrication of active layer. Adv. Energy Mater. 6, 1600372 (2016).

18. Yang, M. et al. Perovskite ink with wide processing window for scalable high-efficiency solar cells. Nat. Energy 2, 17038 (2017).

19. He, M. et al. Meniscus-assisted solution printing of large-grained perovskite films for high-efficiency solar cells. Nat. Commun. 8, 16045 (2017).

20. Hwang, K. et al. Toward large scale roll-to-roll production of fully printed perovskite solar cells. Adv. Mater. 27, 1241-1247 (2015).

21. $\mathrm{Hu}, \mathrm{Q}$. et al. In-situ dynamic observations of perovskite crystallization and microstructure evolution intermediated from $\left[\mathrm{PbI}_{6}\right]$ 4-cage nanoparticles. Nat. Commun. 8, 15688 (2017).

22. Di Giacomo, F. et al. Up-scalable sheet-to-sheet production of high efficiency perovskite module and solar cells on 6-in. substrate using slot die coating. Solar Energy Mater. Solar Cells (in the press).

23. Tang, S. et al. Composition engineering in doctor-blading of perovskite solar cells. Adv. Energy Mater. 7, 1700302 (2017).

24. Ye, F. et al. Low-temperature soft-cover deposition of uniform large-scale perovskite films for high-performance solar cells. Adv. Mater. 29, 1701440 (2017).

25. Li, Y. et al. In situ observation of crystallization of methylammonium lead iodide perovskite from microdroplets. Small 13, 1604125 (2017).

26. Le Berre, M., Chen, Y. \& Baigl, D. From convective assembly to LandauLevich deposition of multilayered phospholipid films of controlled thickness. Langmuir 25, 2554-2557 (2009).

27. Kim, H. et al. Controlled uniform coating from the interplay of Marangoni flows and surface-adsorbed macromolecules. Phys. Rev. Lett. 116 124501 (2016)

28. Zheng, X. et al. Defect passivation in hybrid perovskite solar cells using quaternary ammonium halide anions and cations. Nat. Energy 2, 17102 (2017).

29. Fang, Y. \& Huang, J. Resolving weak light of sub-picowatt per square centimeter by hybrid perovskite photodetectors enabled by noise reduction. Adv. Mater. 27, 2804-2810 (2015).

30. $\mathrm{Bi}, \mathrm{C}$. et al. Non-wetting surface-driven high-aspect-ratio crystalline grain growth for efficient hybrid perovskite solar cells. Nat. Commun. 6, 7747 (2015).

31. McLauchlin, M. L. et al. Evaporative properties and pinning strength of laser-ablated, hydrophilic sites on lotus-leaf-like, nanostructured surfaces. Langmuir 23, 4871-4877 (2007).

32. Milne, A. J. SpringerAmpamp; Amirfazli, A. Autophilic effect: wetting of hydrophobic surfaces by surfactant solutions. Langmuir 26, 4668-4674 (2009).

33. Stoebe, T., Lin, Z., Hill, R. M., Ward, M. D. \& Davis, H. T. Surfactantenhanced spreading. Langmuir 12, 337-344 (1996).

34. Rakocevic, L. et al. Interconnection optimization for highly efficient perovskite modules. IEEE J. Photovolt. 7, 404-408 (2017).

35. Palma, A. L. et al. Laser-patterning engineering for perovskite solar modules with 95\% aperture ratio. IEEE J. Photovolt. 7, 1674-1680 (2017).

36. Moon, S.-J. et al. Laser-scribing patterning for the production of organometallic halide perovskite solar modules. IEEE J. Photovolt. 5, 1087-1092 (2015)

\section{Acknowledgements}

We thank the financial support from Office of Naval Research under award N00014-15-1-2713 and N00014-17-1-2619, and UNC Research Opportunities Initiative. This work was performed in part at the Chapel Hill Analytical and Nanofabrication Laboratory, CHANL, a member of the North Carolina Research Triangle Nanotechnology Network, RTNN, which is supported by the National Science 
Foundation, grant ECCS-1542015, as part of the National Nanotechnology Coordinated Infrastructure, NNCI. We thank Z. Yu and Z. Holman at Arizona State University for their independent measurements of the fabricated perovskite solar modules.

\section{Author contributions}

J.H. and Y.D. conceived the idea and designed the experiments. Y.D. fabricated the perovskite thin films and devices and conducted the characterization. Y.B. and X.Z. synthesized and prepared the raw chemicals for perovskite thin film deposition. Y.D. and Q.W. conducted the perovskite solution droplet experiment. J.Z. helped with the laser ablation process for module fabrication. J.H. and Y.D. wrote the paper, and all authors reviewed the paper.

\section{Competing interests}

The authors declare no competing interests.

\section{Additional information}

Supplementary information is available for this paper at https://doi.org/10.1038/ s41560-018-0153-9.

Reprints and permissions information is available at www.nature.com/reprints. Correspondence and requests for materials should be addressed to J.H.

Publisher's note: Springer Nature remains neutral with regard to jurisdictional claims in published maps and institutional affiliations. 


\section{natureresearch}

\section{Solar Cells Reporting Summary}

Nature Research wishes to improve the reproducibility of the work that we publish. This form is intended for publication with all accepted papers reporting the characterization of photovoltaic devices and provides structure for consistency and transparency in reporting. Some list items might not apply to an individual manuscript, but all fields must be completed for clarity.

For further information on Nature Research policies, including our data availability policy, see Authors \& Referees.

\section{- Experimental design}

\section{Please check: are the following details reported in the manuscript?}

1. Dimensions

Area of the tested solar cells

Method used to determine the device area

2. Current-voltage characterization

Current density-voltage (J-V) plots in both forward and backward direction

Voltage scan conditions

For instance: scan direction, speed, dwell times

Test environment

For instance: characterization temperature, in air or in glove box

Protocol for preconditioning of the device before its characterization

Stability of the J-V characteristic

Verified with time evolution of the maximum power point or with the photocurrent at maximum power point; see ref. 7 for details.

3. Hysteresis or any other unusual behaviour

Description of the unusual behaviour observed during the characterization

Related experimental data

\section{Efficiency}

External quantum efficiency (EQE) or incident photons to current efficiency (IPCE)

A comparison between the integrated response under the standard reference spectrum and the response measure under the simulator

For tandem solar cells, the bias illumination and bias voltage used for each subcell

\section{Calibration}

Light source and reference cell or sensor used for the characterization

Confirmation that the reference cell was calibrated and certified
XYes

$\square$ No

$\square$ Yes

Xno

Хres

$\square$ No

XYes

$\square$ No

Xyes

$\square$ No

$\square$ Yes

¿no

Хyes No

$X$ Yes No

Xyes $\square$ No

$\triangle$ Yes $\square$ No

XYes No

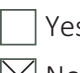
\No

ХYes $\square$ No

XYes $\square$ No
Section "Blade coated perovskite device characterization" \& Supplementary Table 2

It is large $(30-60 \mathrm{~cm} 2)$ so can be simply measured by a ruler or caliper accurately.

Section "Blade coated perovskite device characterization" \& Supplementary Table 2 \& Supplementary Figure 11

Section "Device characterization" \& Supplementary Figure 11

Supplementary Figure 11

No preconditioning, as stated in Section "Device characterization"

Section "Blade coated perovskite device characterization"

Minor hysteresis observed, stated in Section "Blade coated perovskite device characterization"

Figure 5b \& Supplementary Table 2 \& Supplementary Figure 11

Section "Blade coated perovskite device characterization" \& Supplementary Figure 7

Section "Blade coated perovskite device characterization"

Not tandem solar cells

Section "Device characterization"

Section "Device characterization" 
Calculation of spectral mismatch between the reference cell and the devices under test

6. Mask/aperture

Size of the mask/aperture used during testing

Variation of the measured short-circuit current density with the mask/aperture area

\section{Performance certification}

Identity of the independent certification laboratory that confirmed the photovoltaic performance

A copy of any certificate(s)

Provide in Supplementary Information

8. Statistics

Number of solar cells tested

Statistical analysis of the device performance

9. Long-term stability analysis

Type of analysis, bias conditions and environmental conditions

For instance: illumination type, temperature, atmosphere humidity, encapsulation method, preconditioning temperature
Calculation is not reported but we reported all needed information including our device spectrum and the model of Silicon reference cell (Newport 91150V-KG5) in Section "Device characterization" .

$\triangle$ Yes

Section "Blade coated perovskite device characterization" \& Supplementary Table 2

No

$\square$ Yes

The short-circuit current density doesn't vary with the mask/aperture area.

$\triangle$ No

$\triangle$ Yes

By an external decent photovoltaic lab in ASU

No

$\triangle$ Yes

The independent test results have been included as Supplementary Figure 11

No

$\triangle$ Yes

Section "Blade coated perovskite device characterization"

No

$\triangle$ Yes

Supplementary Figure 10

No

$\triangle$ Yes

Section "Blade coated perovskite device characterization" 